\title{
The five-year survival of gastric cancer patients with a focus on its prognostic factors
}

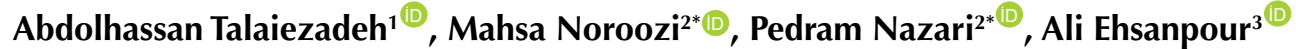 \\ ${ }^{1}$ Cancer Research Center, Ahvaz Jundishapur University of Medical Science, Ahvaz, Iran \\ ${ }^{2}$ Student Research Committee, Ahvaz Jundishapur University of Medical Science, Ahvaz, Iran \\ ${ }^{3}$ Thalassemia and Hemoglobinopathy Research Center, Health Research Institute, Ahvaz Jundishapur University of Medical \\ Sciences, Ahvaz, Iran
}

\section{Correspondence to:}

Pedram Nazari,

Email: nazari.p@ajums.ac.ir and

Mahsa Noroozi,

Email: noroozi.m@ajums.ac.ir

Received: 2 Sep. 2021

Accepted: 3 Oct. 2021

ePublished: 25 Oct. 2021

Keywords: Gastric cancer, Overall survival, Surgery

\begin{abstract}
Introduction: Despite extensive advances in the early diagnosis and treatment of gastric cancer, it is still one of the most common causes of death worldwide. In addition to stage and recurrence, the degree of tumor differentiation affects the survival of patients.

Objectives: The current study was conducted to investigate the prognostic factors and survival rate of patients with gastric cancer.

Patients and Methods: The current prospective study was conducted on patients with gastric cancer from 2010 to 2017 in Imam Khomeini and Apadana hospitals in Ahvaz, Southwest of Iran. For each patient, curative (total or subtotal gastrectomy with D2 lymphadenectomy) or palliative surgery (partial gastrectomy and gastrojejunostomy) were carried out based on clinical indications. Then Cox model was used to analyze factors affecting survival and the Kaplan-Meier test was also utilized to analyze the five-year survival of patients with SPSS version 21.

Results: Around 154 patients with gastric cancer, including 101 males (65.6\%), with a mean age of $62.21 \pm 13.76$ years, were studied. The two- and five-year overall survival (OS) of patients were $57.7 \%$ and $28.5 \%$, respectively (median OS $=28 \mathrm{~m}$ ). Lower stage, well-differentiated tumors, absence of metastasis, lack of recurrence, receiving adjuvant chemotherapy and curative surgery were significantly associated with greater OS $(P<0.05)$.

Conclusion: OS rate of patients with gastric cancer undergoing surgery, especially in the ones with highgrade tumors, metastasis and recurrence, is relatively low, hence careful implementation of screening programs should be considered as the most important preventive strategy.
\end{abstract}

\section{Introduction}

Gastric cancer is one of the most common malignancies worldwide and 782685 deaths occurred by this disease in 2018 (1). Similar to other countries, gastric cancer in Iran has a relatively high incidence and the most important cause of this issue is the late diagnosis of gastric cancers due to delayed presentation and no screening programs (2). According to the Iranian National Population-based Cancer Registry, the agespecific rate incidence of gastric cancer in Khuzestan province in 2014 was 13.52 and 6.32 in males and females, respectively (3). Despite recent advances in prevention, screening and management of gastric cancer patients, the disease has a significant increase in incidence and it is estimated that only $28.3 \%$ of newly diagnosed patients can survive for five years or more after diagnosis (4). According to a recently published metaanalysis, one-two-, three, four and five-year

\begin{abstract}
Key point
The current study showed that two- and five-year overall survival of patients with gastric cancer in Khuzestan province, Southwest of Iran were $57.7 \%$ and $28.5 \%$, respectively (median overall survival $=28$ $\mathrm{m})$. Lower stage, well-differentiated tumors, absence of metastasis, lack of recurrence, receiving adjuvant chemotherapy and curative surgery were significantly associated with greater overall survival.
\end{abstract}

survival rates of patients with gastric cancer in the Iranian population were $52 \%, 31 \%$, $24 \%, 22 \%$ and $15 \%$, respectively (5).

The survival rate of patients with gastric cancer depends on various factors such as age, stage of disease, type of treatment and histopathology, location and degree of tumor differentiation (6). According to the results of the study by Gholizadeh and Malekpour, the mortality rate was directly correlated with the higher stage (7). Among other poor prognostic factors of gastric cancer, more

Copyright $\odot 2022$ The Author(s); Published by Society of Diabetic Nephropathy Prevention. This is an open-access article distributed under the terms of the Creative Commons Attribution License (http://creativecommons.org/licenses/by/4.0), which permits unrestricted use, distribution, and reproduction in any medium, provided the original work is properly cited. 
involvement of lymph nodes (8) and lack of receiving neoadjuvant or adjuvant chemotherapies (9) are other important poor prognostic factors. In addition, some studies showed that radical resection of the tumor with sufficient lymphadenectomy to achieve R0 resection (at least D2 gastrectomy) is another factor influencing the survival of patients with gastric cancer (10). Although the survival rate and prognostic factors of patients with gastric cancer are evaluated in some studies; however, there are controversies and heterogeneities among these studies and poor generalizability of their results to other communities.

\section{Objectives}

We designed the current study to evaluate the five-year overall survival (OS) of patients with gastric cancer and its prognostic factors.

\section{Patients and Methods \\ Study design}

The current prospective study was conducted on all patients with gastric cancer undergoing surgery by a cancer surgery team in Imam Khomeini and Apadana hospitals in Ahvaz, Southwestern Iran from March 2010 to March 2019. These hospitals of Ahvaz are cancer referral centers in Southwest Iran and even some Eastern areas of Iraq and currently of the largest cancer surgery centers in the southwest of Iran.

The inclusion criteria of the current study were all patients with gastric cancer undergoing surgery by the hospital-based surgical team in mentioned hospitals in Ahvaz. Exclusion criteria were patients with major missing data in their records, which were lost to follow-up or were inaccessible and had no interest to participate in the study.

All patients' data were entered on admission into Admiral Software by a trained staff or cancer surgeon. Details of patients' follow-up visits were also entered into the software. Demographic data of all patients including age at diagnosis, gender, tumor location, type of tumor pathology, degree of tumor differentiation, stage of disease, tumor recurrence, type of treatment (surgery, chemotherapy and/or adjuvant or neoadjuvant radiotherapy), type of tumor resection (palliative or curative as D2 gastrectomy or D1 gastrectomy or gastrojejunostomy), and OS were also collected. The survival status of patients was followed up through interval visiting or telephone calls. OS was defined as the time from definitive diagnosis to death or the last follow-up in case of survival.

\section{Statistical analysis}

The qualitative variables were reported in the study as frequency and percentage in each category. Quantitative variables were also measured as mean $\pm \mathrm{SD}$. The fiveyear OS rate and median OS and median survival for each studied variable were also calculated employing the Kaplan-Meier method. The median survival of patients was reported in the two- and five-year survival rates.
Then, the Cox regression model was applied to investigate the factors affecting patients' survival. (11). Data were analyzed with SPSS version 21. $P<0.05$ was considered the level of significance

\section{Results}

A total of 222 patients with gastric cancer undergoing surgery were enrolled in the study of whom, 68 were excluded due to major missing data, lack of access, loss to follow-up and lack of interest to participate in the study. Finally, 154 patients were studied, of whom 101 $(65.6 \%)$ were male. The mean age of the participants was $62.21 \pm 13.76$ years. The two- and five-year OS of the patients was $57.7 \%$ and $28.5 \%$, respectively (median $\mathrm{OS}=28$ months). In addition, by the time of the last followup, 56 (36.4\%) had died (Table 1).

According to Table 2, variables such as age, tumor size, tumor location, receiving neoadjuvant chemotherapy and radiotherapy, totalversus subtotal gastrectomy, pathological variants (intestinal versus diffuse) and presence of signetring cells in pathology had no significant effects on survival rate $(P>0.05)$ (Table 2$)$. In the current study, the strongest prognostic factors for the OS of patients with gastric cancer undergoing surgery were metastasis $(\mathrm{HR}=3.47$ (95\% CI: $1.93-6.22)$, tumor stage (HR stage $4 /$ stage $1=4.61$ (95\% CI: 1.77-12.01), tumor recurrence $(\mathrm{HR}=1.70(95 \%$ $\mathrm{CI}: 1.15-2.56)$ and palliative resection $(\mathrm{HR}=3.70(95 \%$ CI: 2.05-6.67) (Table 2 and Figures 1A-1D). According to Table 2, other factors influencing patient survival were lymph node involvement, adjuvant chemotherapy, and tumor differentiation $(P<0.05)$. Hence, patients with N3 (regional lymph nodes) involvement were 3.01 times more likely to die than the ones without lymph node involvement (95\% CI: 0.89-10.12).

In addition, patients receiving adjuvant chemotherapy were 0.60 times less likely to die than other patients (95\% CI: 0.40-0.91). Moreover, well-differentiated gastric tumors had longer survival than other conditions and compared to poor differentiation had the lowest survival rate (HR poor/well- differentiation=3.66 (95\% CI: 1.07 12.31).

Table 1. Survival rate in gastric cancer patients

\begin{tabular}{lll}
\hline Outcome & Number (154) & Percent \\
\hline Relapse & & \\
\hline Yes & 45 & 29.23 \\
No & 109 & 70.77 \\
Death & & 36.4 \\
\hline Yes & 56 & 63.6 \\
No & 98 & \\
Overall survival & \\
Mean & 57.84 months $(\mathrm{Cl} 95 \%: 46.45-69.24)$ \\
Median & 28.00 months $(\mathrm{Cl} 95 \%: 22.16-33.83)$ \\
2 years & 57.7 \\
5 years & 28.5 \\
\hline
\end{tabular}


Table 2. Estimation of the coefficient of Cox proportional hazard model and hazard ratio for gastric cancer patients

\begin{tabular}{|c|c|c|c|c|c|}
\hline Covariate & 5 years OS $(\%)$ & Median OS (month) & Hazard ratio & $95 \% \mathrm{Cl}$ & $P$ value \\
\hline Gender (male/female) & $32 / 20$ & $30 / 25$ & 0.84 & $0.56-1.9227$ & 0.40 \\
\hline Age (year) & - & - & 1.007 & $0.99-1.02$ & 0.58 \\
\hline Tumor site (proximal/distal) & $29 / 40$ & $32 / 40$ & 0.80 & $0.47-1.38$ & 0.43 \\
\hline Tumor size & & - & - & - & 0.58 \\
\hline T1 & 50 & 43 & - & - & - \\
\hline $\mathrm{T} 2$ & 46 & 38 & 1.14 & $0.24-5.55$ & 0.86 \\
\hline $\mathrm{T} 3$ & 37 & 25 & 1.74 & $0.3-7.59$ & 0.46 \\
\hline $\mathrm{T} 4$ & 30 & 20 & 2.24 & $0.43-11.59$ & 0.33 \\
\hline Lymph node & & & & & 0.12 \\
\hline No & 62 & - & & - & \\
\hline N1 & 35 & 32 & 2.21 & $0.89-5.50$ & 0.08 \\
\hline N2 & 25 & 23 & 2.83 & $1.02-7.87$ & 0.04 \\
\hline N3 & 20 & 21 & 3.01 & $0.89-10.12$ & 0.07 \\
\hline Metastasis (Yes/No) & $9 / 54$ & $20 /-$ & 3.47 & $1.93-6.22$ & 0.000 \\
\hline Stage & & & & & 0.001 \\
\hline 1 & 53 & - & & - & \\
\hline II & 50 & - & 1.48 & $0.46-4.66$ & 0.50 \\
\hline III & 43 & - & 1.69 & $0.53-5.36$ & 0.36 \\
\hline IV & 1 & 20 & 4.61 & $1.77-12.01$ & 0.002 \\
\hline Relapse (Yes/No) & $15 / 35$ & $23 / 35$ & 1.90 & $1.27-2.86$ & 0.003 \\
\hline Radiotherapy (Yes/No) & $20 / 31$ & $31 / 27$ & 0.99 & $0.61-1.59$ & 0.97 \\
\hline Adjuant chemotherapy (Yes/No) & $35 / 20$ & $36 / 25$ & 0.60 & $0.40-0.91$ & 0.01 \\
\hline Neoadjuant chemotherapy (Yes/No) & $22 / 39$ & $27 / 31$ & 1.37 & $0.90-2.06$ & 0.14 \\
\hline Surgery kind (total gastrectomy/subtotal gastrectomy) & $23 / 36$ & $24 / 35$ & 1.28 & $0.80-2.03$ & 0.29 \\
\hline Resection (palliative/curative) & $10 / 50$ & 20/- & 3.70 & $2.05-6.67$ & 0.000 \\
\hline Differentiation & & & & & 0.17 \\
\hline Well & 72 & 80 & - & - & - \\
\hline Moderate & 40 & 25 & 2.44 & $0.64-9.22$ & 0.18 \\
\hline Poor & 22 & 17 & 3.63 & $1.07-12.31$ & 0.03 \\
\hline Unknown & 24 & 27 & 2.97 & $0.93-9.49$ & 0.06 \\
\hline Pathological variants (intestinal/diffuse) & $32 / 23$ & $22 / 24$ & 0.91 & $0.44-1.90$ & 0.80 \\
\hline Signet ring (Yes/No) & $16 / 31$ & $25 / 29$ & 1.36 & $0.77-2.41$ & 0.28 \\
\hline
\end{tabular}

\section{Discussion}

Gastric cancer is one of the deadliest cancers with a relatively high prevalence in Iran and is the second leading cause of cancer death worldwide (12). The present research was one of the largest prospective studies on the survival of patients with gastric cancer in Southwestern Iran.

Our results indicated that the five-year survival rate of gastric cancer was $28.5 \%$ and the recurrence rate was 20.1\%. Similar to our findings, Nagaich and Sharma (4), Soroush (13), Noorkojuri et al (14), Moghimi-Dehkordi et al (15) and Baeradeh et al (16)demonstrated 28\%, 31\%, $30 \%, 29 \%$ and $25 \%$ of patients with gastric cancer survive at least five years, respectively. Contrary to our results, Samadi et al, Mehrabian et al, Biglarian et al, Ghadimi et al and Veisani \& Delpisheh (17-21) reported the fivesurvival rate of gastric cancer $8 \%, 15 \%, 17 \%, 15 \%$ and $6 \%$. It seems that the main cause of the controversy in the survival of gastric cancer in different studies is due to their heterogeneity such as different data gathering, varieties in enrolled baseline patient characteristics (different races, stages, tumor grades, histopathologic subtypes and treatments), the year and sample size of studies (5) and the study protocol and chronology (prospective versus retrospective). For example, in the study of AkhondiMeybodi et al (6) $50.5 \%$ of the patients had stage 4 cancer while it was $24.5 \%$ in our study and it can be a potential explanation for better survival in our investigation $(28.5 \%$ versus $18 \%$ ).

The present study showed that palliative and curative resections were associated with a five-year survival of $9 \%$ and $50 \%$, respectively. Consistent with the present study results, Zhang et al showed that the five-year OS of patients undergoing palliative and curative surgeries was $7.8 \%$ and $51.2 \%$, respectively. In addition, patients undergoing surgical resection had a five-year OS of 31.1\% (22). In another study by Káposztás et al, the five-year 
(A)

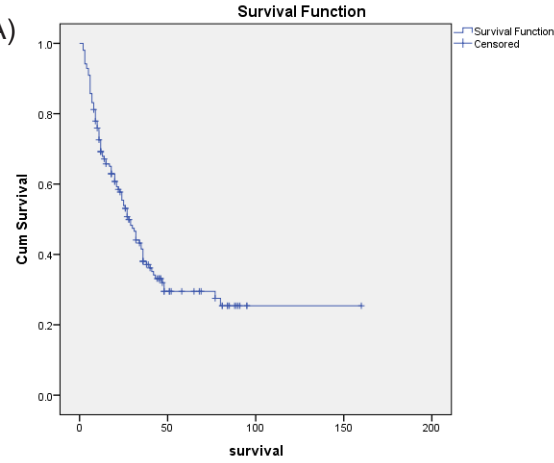

(C)

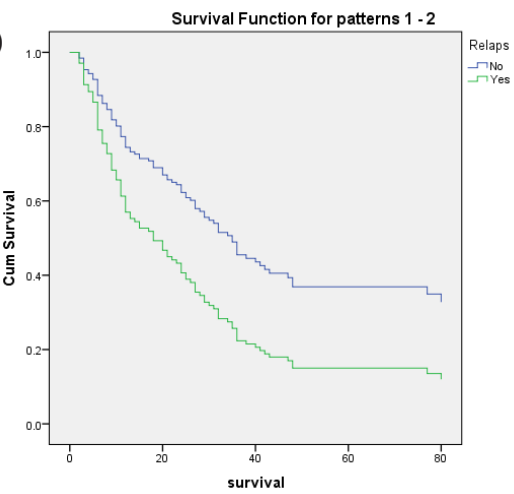

(B)
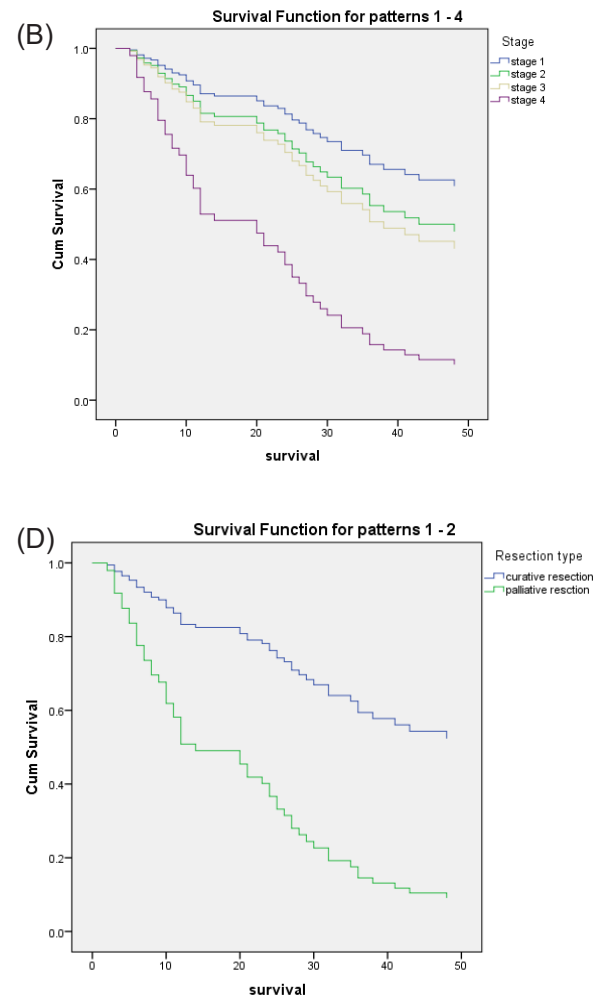

Figure 1. (A) Kaplan-Meier estimates of overall survival in gastric cancer patients. Kaplan-Meier estimates of overall survival probability based on (B) tumor staging, (C) tumor recurrence and (D) resection type.

OS of patients with gastric cancer undergoing curative resection was $69.5 \%$, which was similar to the results of the current study (10).

Our study showed that some factors such as disease stage, degree of differentiation, metastasis, recurrence and type of treatment had a significant effect on patients' survival. The results of the study by Modarres et al on patients with gastric cancer who underwent surgery showed that gender, age, location and type of tumor, lymph nodes involvement, metastasis status and type of treatment had not a significant correlation with survival, whereas cancer staging and surgery method was a significant prognostic factor in gastric cancer outcome (23). Additionally, Maroufizadeh et al consistent with the current study findings, showed that metastasis had a significant effect on survival (24). Another study showed that age, stage of disease, type of treatment and histopathological variant were important prognostic factors in the survival of patients with gastric cancer (6). Similar to the current study, Nagaich et al (4) showed that the survival rate of patients with well-differentiated tumors was higher than other tumor differentiation subtypes. In other studies, independent risk factors associated with survival included disease stage, tumor location (upper third), degree of differentiation, metastasis, type of resection (palliative or curative), lymph node involvement ( $\mathrm{N}$ stage) and depth of tumor invasion $(10,22)$.

Our investigation showed that patients receiving adjuvant chemotherapy had a higher survival rate than those who did not. In agreement with the results of the present study, some studies showed improved survival of patients with gastric cancer after receiving adjuvant and neoadjuvant chemotherapy $(25,26)$. In addition, studies by the Medical Research Council Adjuvant Gastric Infusional Chemotherapy (MAGIC) indicated that chemotherapy and chemoradiotherapy along with surgery can significantly increase patients' survival; these techniques are utilized as standard treatments in advanced stages of the disease (27).

\section{Conclusion}

The findings of current study indicate that the OS rate of patients with gastric cancer who underwent surgery is generally low (five-year OS=28.5\%); it was significantly correlated with the type of resection (palliative/curative), stage of disease, metastasis and tumor recurrence, lymph node involvement ( $\mathrm{N}$ stage), the type of adjuvant therapy, and tumor grading.

\section{Limitations of the study}

The study limitations included small sample size, only enrollment of patients undergoing surgery, and negligence of other potential variables probably involved in survival, including other risk factors such as underlying diseases, nutritional habits, alcohol, tobacco and opioid use, and lack of assessment in terms of $\mathrm{H}$. pylori infection.

It is recommended to conduct an extensive study 
using international cancer registry data, relying on different demographic, environmental, clinical and histopathological, and therapeutic factors to better understand the factors contributing to the survival of gastric cancer patients. It is suggested that the results of this study be compared with patients who are sporadically operated in non-specialist cancer centers and by general surgeons. We believe that training and experience in gastric cancer surgery can produce better results.

\section{Authors' contribution}

AT and PN designed the protocol of study. AT, MN and PN participated in the implementation study. All authors worked for data analysis and manuscript writing

\section{Conflicts of interest}

The authors declared no conflicts of interest.

\section{Ethical issues}

The study protocol was approved by the Ethics Committee of Ahvaz Jundishapur University of Medical Sciences (IR.AJUMS.HGOLESTAN. REC.1399.007) and written informed voluntary consent was also obtained from all subjects according to the Declaration of Helsinki principles. This study was extracted from M.D thesis of Mahsa Noroozi at this university (Thesis \# CRC-9906). Additionally, ethical issues (including plagiarism, data fabrication and double publication) were completely observed by the authors.

\section{Funding/Support}

This study was granted by Cancer Research Center, Ahvaz Jundishapur University of Medical Science, Ahvaz, Iran (Grant\#CRC-9906).

\section{References}

1. Thrift AP, El-Serag HB. Burden of gastric cancer. Clin Gastroenterol Hepatol. 2020;18:534-542. doi: 10.1016/j.cgh.2019.07.045.

2. Ahmadzadeh A, Seyedian SS, Valizadeh A, Soleimani M, Nazari P, Hamidi $\mathrm{H}$. The efficacy and safety of modified docetaxel, cisplatin, and 5-fluorouracil vs. epirubicin, oxaliplatin, and capecitabine regimen in the advanced gastric cancer: A randomized controlled clinical trial. Asian Pac J Cancer Prev. 2020;21:727-32. doi: 10.31557/APJCP.2020.21.3.727.

3. Roshandel G, Ghanbari-Motlagh A, Partovipour E, Salavati F, Hasanpour-Heidari S, Mohammadi G, et al. Cancer incidence in Iran in 2014: results of the Iranian National Populationbased Cancer Registry. Cancer Epidemiol. 2019;61:50-58. doi: 10.1016/j.canep.2019.05.009.

4. Nagaich N, Sharma R. Gastric cancer-an update. J Tumor Med Prev. 2018;2:555597. doi: 10.19080/JTMP.2018.02.

5. Veisani $Y$, Delpisheh A. Survival rate of gastric cancer in Iran; a systematic review and meta-analysis. Gastroenterol Hepatol Bed Bench. 2016;9:78.

6. Akhondi-Meybodi M, Ghane M, Akhondi-Meybodi S, Dashti G. Five-year survival rate for gastric cancer in Yazd Province, Central Iran, from 2001 to 2008. Middle East J Dig Dis. 2017;9:39-48. doi: 10.15171/mejdd.2016.50.

7. Gholizadeh B, Malekpour N. Effective factors on 3-year mortality of operated gastric cancer at Modarres Hospital during 5 years. J Anal Res Clin Med. 2017;5:58-61. doi: 10.15171/jarcm.2017.011

8. Zhou C-M, Wang Y, Ye H-T, Yan S, Ji M, Liu P, et al. Machine learning predicts lymph node metastasis of poorly differentiatedtype intramucosal gastric cancer. Sci Rep. 2021;11:1300. doi: 10.1038/s41598-020-80582-w.

9. Wu F, Hong J, Du N, Wang Y, Chen J, He Y, et al. Long-term outcomes of neoadjuvant chemotherapy in locally advanced gastric cancer/esophagogastric junction cancer: a systematic review and meta-analysis. Anticancer Agents Med Chem. 2021. doi: 10.2174/1871520621666210315091932.
10. Káposztás Z, Kalmár K, Cseke L, Illényi L, Kelemen D, Horváth ÖP. Prognostic factors in the surgical treatment of gastric cancer-10 years experience. Magy Seb. 2007;60:71-8. doi: 10.1556/ MaSeb.60.2007.2.2.

11. Pourhoseingholi MA, Hajizadeh E, Moghimi Dehkordi B, Safaee A, Abadi A, Zali MR. Comparing Cox regression and parametric models for survival of patients with gastric carcinoma. Asian Pac J Cancer Prev. 2007;8:412.

12. Aurello P, Berardi G, Tierno SM, Vinciguerra GLR, Socciarelli F, Laracca GG, et al. Influence of perineural invasion in predicting overall survival and disease-free survival in patients with locally advanced gastric cancer. Am J Surg. 2017;213:748-53. doi: 10.1016/j.amjsurg.2016.05.022.

13. Soroush A. Surgical outcome in patients with gastrointestinal malignancies; a report from a large referral hospital, 2008-2010. Middle East J Dig Dis. 2013;5:201.

14. Noorkojuri H, Hajizadeh E, Baghestani A, Pourhoseingholi M. Application of smoothing methods for determining of the effecting factors on the survival rate of gastric cancer patients. Iran Red Crescent Med J. 2013;15:166-72. doi: 10.5812/ircmj.8649.

15. Moghimi-Dehkordi B, Safaee A, Zali MR. Survival rates and prognosis of gastric cancer using an actuarial life-table method. Asian Pac J Cancer Prev. 2008;9:317-21.

16. Baeradeh NA, Lotfi MH, Fallahzadeh H, Kargar S, Salman RH. Survival rate of patients with stomach cancer and its effective factors in Yazd Province. Journal of Community Health Research. 2015;3:278-87.

17. Samadi F, Nasr ED, Sajadi A, DERAKHSHAN M, Malekzadeh R, Fuladi $R$, et al. Survival rate of gastric and esophageal cancers in Ardabil province, North-West of Iran. Arch Iran Med. 2007;10:3237.

18. Mehrabian A, Esna-Ashari F, Zham H, Hadizadeh M, Bohlooli M Khayamzadeh $\mathrm{M}$, et al. Gastric cancer prevalence, according to survival data in Iran (National Study-2007). Iran J Public Health. 2010;39:27-31.

19. Biglarian A, Hajizadeh E, Kazemnejad A, Zali M. Application of artificial neural network in predicting the survival rate of gastric cancer patients. Iran J Public Health. 2011;40:80-6.

20. Ghadimi M, Mahmoodi M, Mohammad K, Zeraati H, Rasouli M, Sheikhfathollahi M. Family history of the cancer on the survival of the patients with gastrointestinal cancer in northern Iran, using frailty models. BMC Gastroenterol. 2011;11:104. doi: 10.1186/1471-230X-11-104.

21. Veisani Y, Delpisheh A, Sayehmiri K, Rahimi E. Demographic and histological predictors of survival in patients with gastric and esophageal carcinoma. Iran Red Crescent Med J. 2013;15:54753. doi: 10.5812/ircmj.11847.

22. Zhang Y-F, Shi J, Yu H-P, Feng A-N, Fan X-S, Lauwers GY, et al. Factors predicting survival in patients with proximal gastric carcinoma involving the esophagus. World J Gastroenterol. 2012;18:3602-9. doi: 10.3748/wjg.v18.i27.3602.

23. Modarres S-R, Gholizadeh-Pasha A, Khatibi S, Siadati S. Estimating postoperative survival rate of gastric cancer patients and its effective factors in Babol, northern Iran: 2006-2011. Caspian J Intern Med. 2017;8:190-5. doi: 10.22088/cjim.8.3.190.

24. Maroufizadeh S, Hajizadeh E, Baghestani AR, Fatemi SR Determining the postoperative survival in patients with gastric cancer and the associated factors using Cox and Lin-Ying additive hazards models. J Arak Uni Med Sci. 2012;15:84-92.

25. Oblak I, Velenik V, Anderluh F, Strojan P. Results of adjuvant radiochemotherapy for gastric adenocarcinoma in Slovenia. Eur J Surg Oncol. 2007;33:982-7. doi: 10.1016/j.ejso.2006.12.012.

26. Luo W, Zhang H, Zhao Y, Wang L, Qi L, Ran J, et al. A retrospective study on intensity-modulated radiation therapy combined with chemotherapy after D2 radical surgery for gastric carcinoma. Mol Clin Oncol. 2016;4:740-8. doi: 10.3892/mco.2016.804.

27. Ahmad S, Hanna N. Treatment of resectable gastric cancer: an update on the role of radiation and chemotherapy. Clin Surg. $2016 ; 1: 1223$ 